\title{
Anterior sacral meningocele repair assisted by intraoperative intrathecal fluorescence and 3D printing model: illustrative case
}

\author{
${ }^{*} Y u-C h a i n g$ Yeh, MD, ${ }^{1}$ Ya-Jui Lin, MD, ${ }^{1,2}$ Chih-Hua Yeh, MD, ${ }^{3,4}$ Pao-Shiu Hsieh, MD, ${ }^{5}$ and Chieh-Tsai Wu, MD ${ }^{1}$ \\ ${ }^{1}$ Department of Neurosurgery, Chang-Gung Memorial Hospital, Chang Gung University College of Medicine, Taoyuan City, Taiwan; ${ }^{2}$ The Graduate Institute of Biomedical \\ Sciences and ${ }^{4}$ School of Medicine, Chang Gung University, Taoyuan City, Taiwan; and ${ }^{3}$ Department of Neuroradiology and ${ }^{5}$ Division of Colon and Rectal Surgery, Chang Gung \\ Memorial Hospital, Guishan Township, Taoyuan City, Taiwan
}

\begin{abstract}
BACKGROUND Marfan syndrome is rarely accompanied by anterior sacral meningocele (ASM) resulting from erosion of the sacrum by dural ectasia. ASM may induce symptoms due to severe mass effects. ASM may also mimic ovarian cysts, and the risk of cerebrospinal fluid (CSF) leakage is high if spontaneous rupture of the cyst occurs. In this study, the authors presented a rare case of ASM with iatrogenic CSF leakage in a 34-year-old woman with suspected Marfan syndrome.
\end{abstract}

OBSERVATIONS The patient initially presented with a giant ASM that was first misdiagnosed as an ovarian cyst. Previously, it had been partially resected, which was followed by iatrogenic CSF leakage. Symptoms of intracranial hypotension, including postural headache and dizziness, developed within 1 month. Brain magnetic resonance imaging (MRI) showed pituitary enlargement, bilateral subdural effusion, and tonsillar herniation.

Preoperative computed tomography myelography provided three-dimensional (3D) examination of the deformed sacrum and CSF leakage site. Transabdominal approaches led to primary repair, and repair of the meningocele was achieved by intraoperative fluorescein fluorescence and 3D printed model-guided polymethyl methacrylate bone cement reconstruction. No CSF leakage or recurrent ASM was found at the 1.5-year follow-up visit.

LESSONS Intraoperative intrathecal fluorescence and 3D-printed models are useful for ASM repair. Preoperative MRI is helpful for differentiating ASM from other causes of a huge pelvic mass, including ovarian cyst.

https://thejns.org/doi/abs/10.3171/CASE20159

KEYWORDS anterior sacral meningocele; ASM; cerebrospinal fluid leak; intracranial hypotension; Marfan syndrome; 3D printing; intrathecal fluorescein

Anterior sacral meningocele (ASM) is a type of meningocele described as the cystic portion with anterior protrusion into the sacral region, usually associated with a sacral defect. Prevalence of the disease is still undetermined because such cases are extremely rare. An ASM is often associated with connective tissue disorders such as Marfan syndrome, Loeys-Dietz syndrome, and neurofibromatosis type I. $^{1-3}$ Marfan syndrome is an autosomal dominant disorder, with a prevalence of 1 in 5000 people. $^{4,5}$ Dural ectasia is a common feature of Marfan syndrome and is found in up to $92 \%$ of patients. ${ }^{6}$ It is defined as the ballooning or widening of the thecal sac, typically found in the sacral spine region, where cerebrospinal fluid (CSF) pressure is greatest. ${ }^{7,8}$ The most severe form of dural ectasia presents as an ASM with associated thinning of the sacral cortex and expansion into the retroperitoneum and pelvis. ${ }^{9}$

Although an ASM is usually asymptomatic, associated symptoms sometimes include radiculopathy, constipation, urinary obstruction, and postural headaches. Several severe complications, such as obstructive hydronephrosis and obstructive renal failure, are also reported. Treatment options include clinical observation and surgical intervention for huge or symptomatic lesions. ${ }^{10}$ Herein, we report on a patient with Marfan syndrome that presented as a giant ASM with

\footnotetext{
ABBREVIATIONS 3D = three dimensional; ASM = anterior sacral meningocele; CSF = cerebrospinal fluid; CT = computed tomography; ITF = intrathecal fluorescein; $\mathrm{MRI}=$ magnetic resonance imaging; PMMA = polymethyl methacrylate.

INCLUDE WHEN CITING Published May 17, 2021; DOI: 10.3171/CASE20159.

SUBMITTED December 21, 2020. ACCEPTED January 25, 2021.

${ }^{*}$ Y.C.Y. and Y.J.L. share first authorship of this work.

(C) 2021 The authors, CC BY-NC-ND 4.0 (http://creativecommons.org/licenses/by-nc-nd/4.0/).
} 
iatrogenic CSF leakage, and we address diagnosis, imaging results, and surgical management.

\section{Illustrative Case}

\section{History and Examination}

A 34-year-old woman was transferred to our emergency department after noticing increased abdominal girth in the previous 6 months. Abdominal echo examination at another institution revealed a huge retroperitoneal cyst with suspected ovarian origin on which a gynecologist performed a laparotomy for partial excision of the cyst. However, postural headache, dizziness, and blurred vision occurred gradually 1 month before her visit to our hospital. Brain computed tomography (CT) and magnetic resonance imaging (MRI) revealed pituitary enlargement (Fig. 1A). After she transferred to our hospital, hormone studies were conducted; all results were within normal range. Upon reviewing her brain images, bilateral thin subdural effusion (Fig. 1B) and tonsillar herniation (Fig. 1A) were found. As a result, the symptoms and MRI findings were compatible with intracranial hypotension. Whole-spine MRI showed diffuse dural ectasia (Fig. 2A), one anterior meningocele with protrusion from the left S1 foramen, and suspected CSF leakage (Fig. 2B). CT myelography was performed to confirm the leakage site, and it revealed extrathecal contrast medium leakage from the left $\mathrm{S} 1$ foramen into the pelvic cavity (Fig. $2 \mathrm{C}$ and D). These findings were compatible with the intracranial hypotension symptoms and her Marfan-like appearance (tall stature). The ASM was originally misdiagnosed as an ovarian cyst and was then resected partially without perfect repair, resulting in iatrogenic CSF leakage.

\section{Surgical Planning and 3D Printing Workflow}

Importing Data Into the Workstation and Initial Preparation

A three-dimensional (3D) CT scan of the pelvis was obtained for preoperative planning using a multislice CT scanner (GE BrightSpeed 16, GE Healthcare) with 0.8-mm intervals. The Digital Imaging and Communications in Medicine format for CT data was imported to image processing software for 3D design and modeling (MIMICS 17.0, Materialise) in an MSI G63VR Stealth Pro laptop with extended graphics card (NVIDIA GTX1060). Manual thresholding (minimum 226 Hounsfield unit, maximum 4,000 Hounsfield unit) was performed to optimize bone intensity from soft tissue components, and a $3 \mathrm{D}$ data set of the bony pelvis was produced (Fig. $3 \mathrm{~A}$ ).
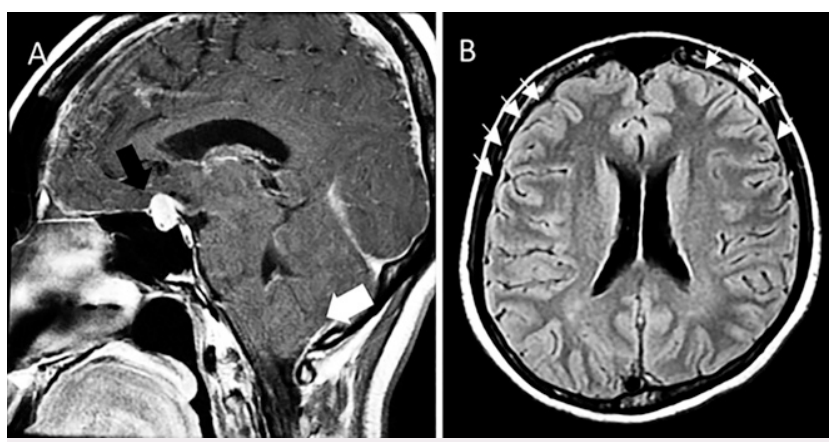

FIG. 1. Preoperative brain MRI. A: Sagittal view shows pituitary enlargement (black arrow) and tonsillar herniation (white arrow). B: Bilateral thin subdural effusion (white arrows) on axial view.
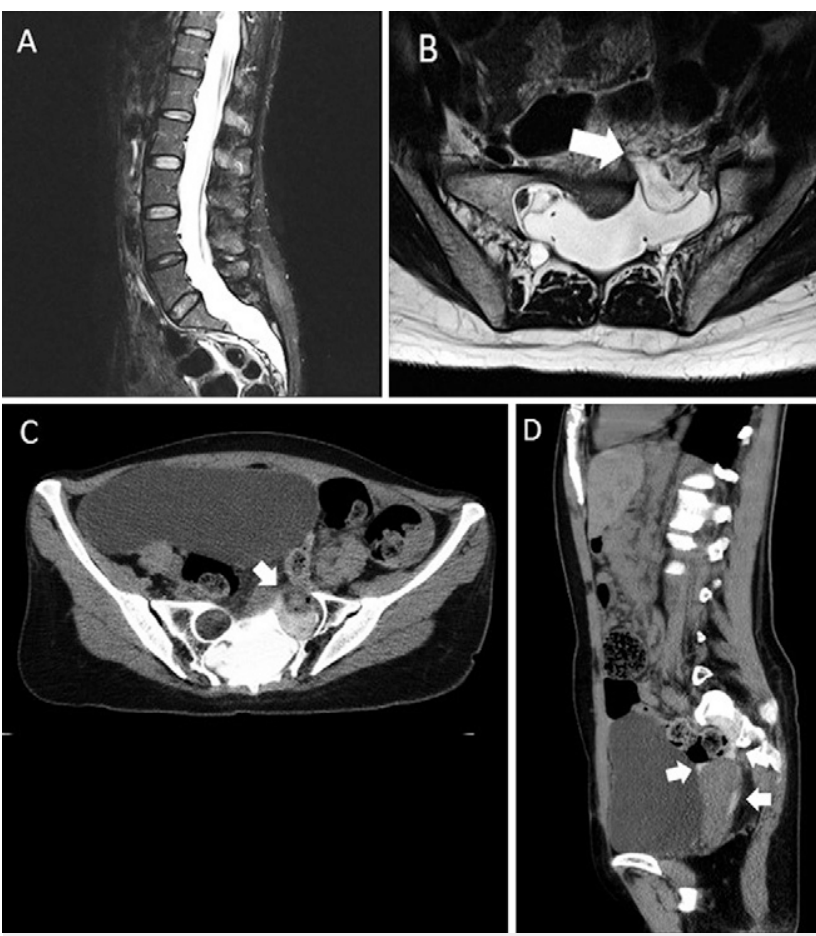

FIG. 2. Lumbar MRI shows dural ectasia (A) and an anterior meningocele from the left S1 foramen and suspected CSF leak (B, white arrow). CT myelography shows extrathecal contrast medium leakage (white arrows) into the pelvic cavity on axial (C) and sagittal (D) views

Incorporating the Surgical Plan Into a 3D-Printed Model

The virtual 3D model was checked on the computer to evaluate the body deformity of the meningocele. It was outputted to a realsize 3D-printed model using a medical-grade 3D printer (Objet30, Stratasys) with biocompatible material (MED610, Stratasys) (Fig. 3B). This 3D model can be used by neurosurgeons and colorectal surgeons to communicate before and during surgery. It can help a surgeon dissect the bony defect of the meningocele and guide the manufacture of a polymethyl methacrylate (PMMA) flap (Fig. $3 \mathrm{C}$ ) to cover the repaired meningeal layer and prevent it from herniating into the pelvic cavity.

\section{Surgical Procedure}

Because of the intracranial hypotension and persistent CSF leakage, surgical intervention was indicated to repair the meningocele and prevent further CSF leakage. To locate the leakage site and to confirm no persistent CSF leakage after repair, low-dose intrathecal fluorescein (ITF) injection (10 mL CSF withdrawn from the patient mixed with $0.25 \mathrm{~mL} 10 \%$ fluorescein solution, Alcon Research Ltd.) was given via lumbar drain with slow infusion for 30 minutes after anesthesia. Then laparotomy was performed by the colorectal surgeon to approach the leakage site. Obvious fluorescent green fluid was identified during the approach to and dissection around the meningocele (Fig. 4A). After primary repair of the defect of the cystic wall, the absence of fluorescent green CSF leakage was confirmed under microscopic inspection. To prevent meningocele recurrence, the bony defect was sealed with a prefabricated PMMA 

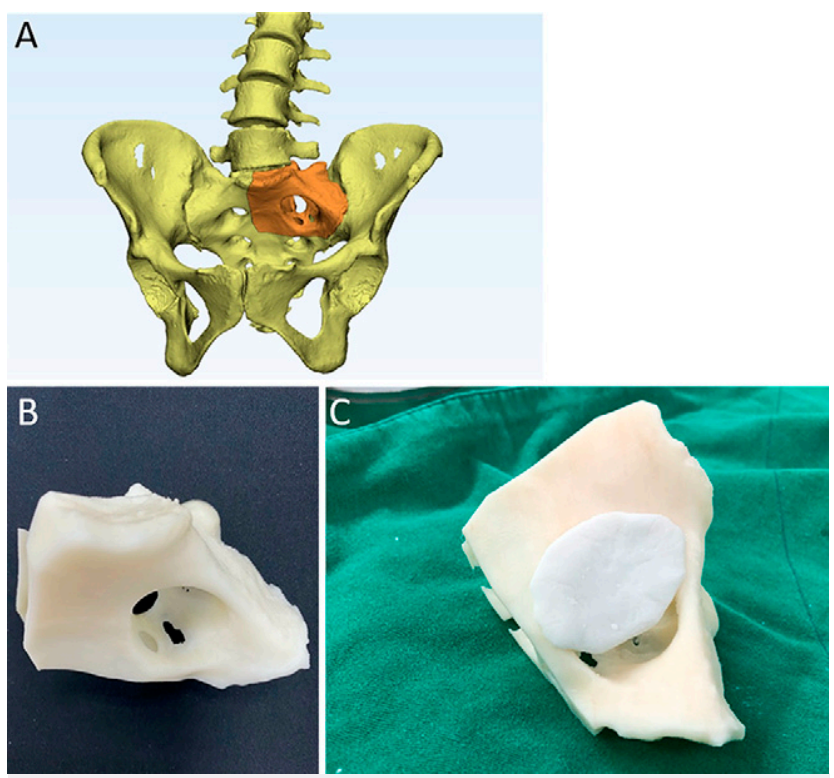

FIG. 3. Virtual 3D presentation of the lesion (A), 3D-printed model of the involved pelvis (B), and 3D-printed model used intraoperatively for PMMA flap production (C).

flap according to the 3D printing model (Fig. 4B). The S1 nerve root was carefully dissected and protected during surgery. Simultaneously, we used one PMMA flap to cover the repaired meningeal sac to prevent it from herniating into the pelvic cavity, and we reserved the space for the S1 nerve root. There was no S1 deficit before or after surgery. Finally, DuraSeal Cranial Sealant System 5 $\mathrm{mL}$ (Integra LifeSciences) was sprayed on the dura and PMMA flap (Fig. 4C), and the omental flap was covered (Fig. 4D).

\section{Postoperative Course}

After the operation, symptoms of intracranial hypotension subsided. At the 9-month postoperative follow-up, lumbar spine MRI showed no recurrence of meningocele or CSF leakage (Fig. 5A and B). At the 3-month postoperative follow-up, brain MRI showed regression of pituitary enlargement, bilateral subdural effusion, and tonsillar herniation (Fig. 5C and D).

\section{Discussion}

\section{Observations}

Dural ectasias from a weakening or expanding of the dura are well-recognized sequelae of Marfan syndrome. They enlarge with CSF pulsations and eventually erode through the vertebral body. ${ }^{11,12}$ ASMs are defined as dural ectasias that expand through bony defects in the sacrum, leading to subsequent herniation of the meninges and occasionally the neural contents. Although the prevalence of ASMs in Marfan syndrome is unknown, dural ectasias are known to occur with high frequency (up to $89 \%$ ) in this population., ${ }^{5,13}$ ASMs are likely to remain asymptomatic until they reach a considerable size. As with any pelvic mass, ultrasound is the first-line imaging modality for ASMs. However, it can be difficult to delineate the origin and extent of an ASM by using ultrasound alone, so further CT or MRI is often needed. ${ }^{2}$ In this case, misdiagnosis as an ovarian cyst
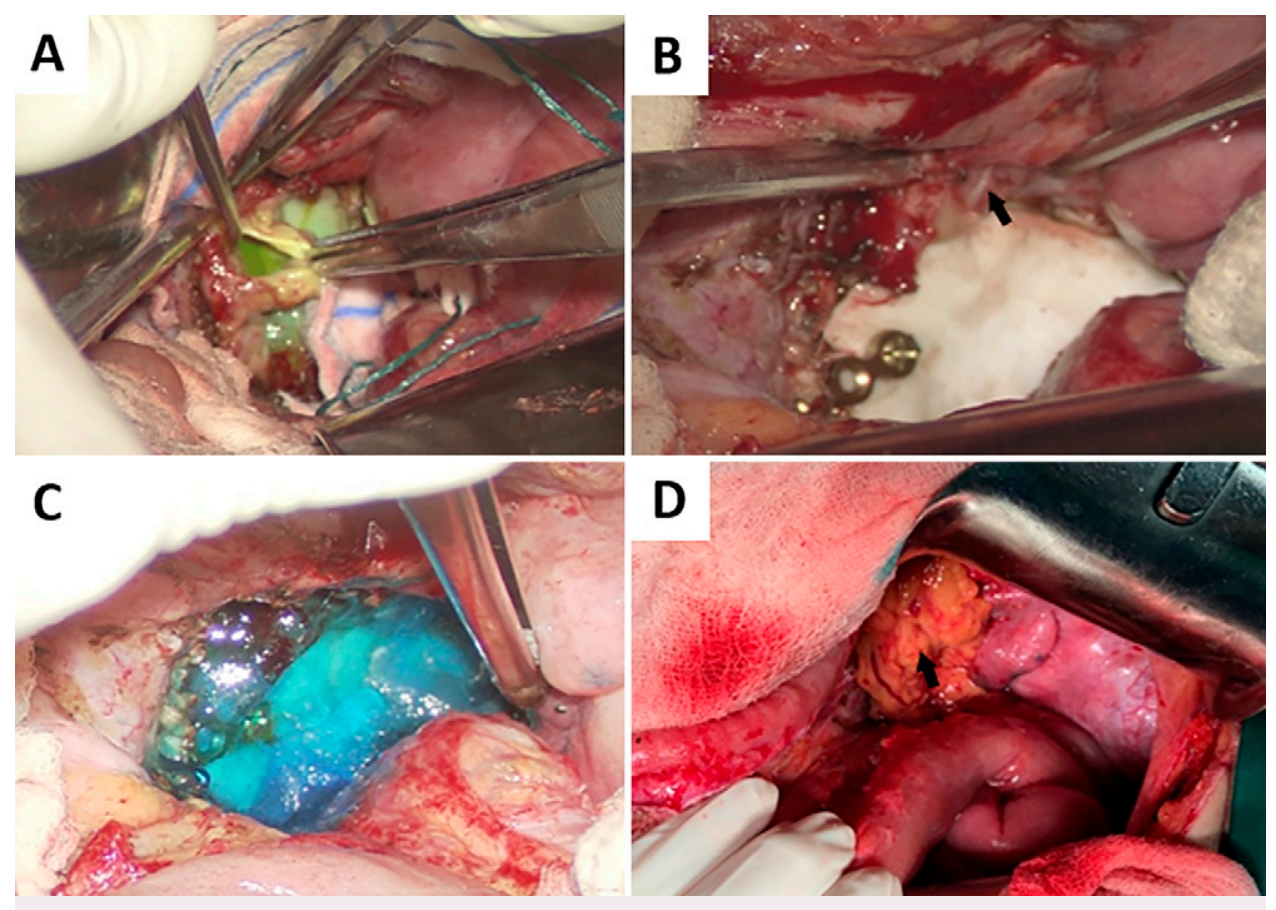

FIG. 4. Intraoperative images. A: Rupture site was identified and filled with fluorescent green CSF. B: Fixed PMMA flap with titanium screws and protected S1 nerve root (black arrow). C: Spray DuraSeal. D: Cover omental flap (black arrow). 

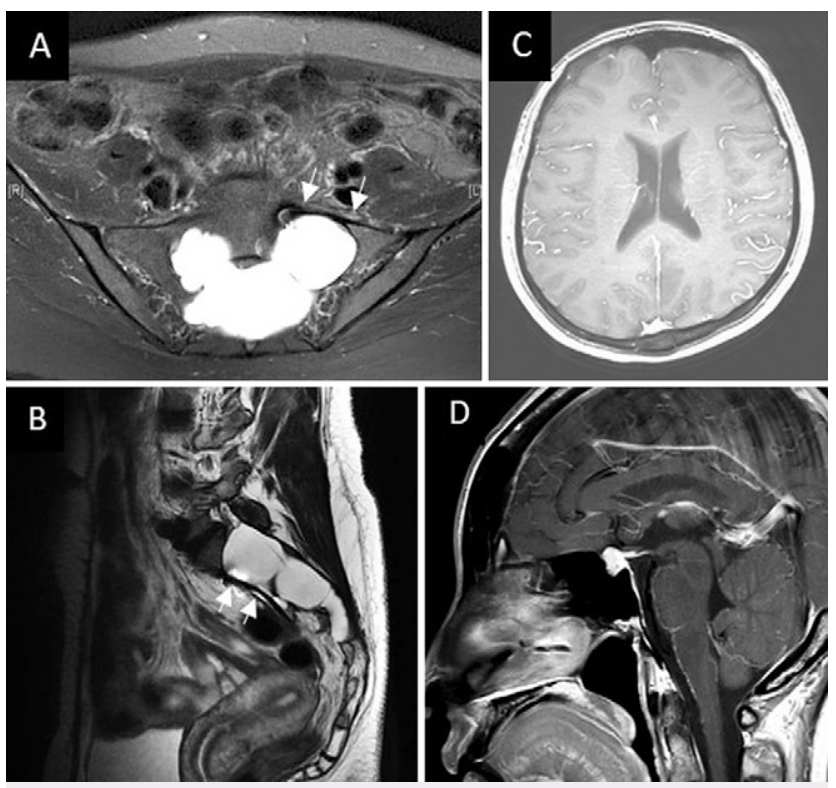

FIG. 5. Axial (A) and sagittal (B) lumbar spine MRI (9 months postoperatively) shows no recurrent ASM and well-positioned bone cement (white arrows) to prevent bulging of dural ectasia. Brain MRI (3 months postoperatively) reveals subsided bilateral subdural effusion (axial, $\mathbf{C}$ ) and decreased enlargement of the pituitary gland and tonsillar herniation (sagittal, D).

occurred because of the use of ultrasound alone without additional $\mathrm{CT}$ or MRI studies. Also, partial cyst resection without closure of the cyst wall was the cause of iatrogenic CSF leakage. Patients with any huge pelvic mass should undergo advanced imaging studies (CT or MRI).

The case discussed here resulted from misdiagnosis as an ovarian cyst and partial cyst resection without prompt repair for almost 1 month. It may be difficult to recognize small CSF leaks because CSF is translucent and the operative field is often blurred with small pools of blood or surrounding adhesion tissue. Because the sacral region is the location of greatest CSF pressure, it is possible for leakage to persist, and it is difficult to achieve spontaneous healing without complete repair. ITF is a useful method by which to identify a leakage site and confirm that no more leakage persists after repair. ${ }^{14}$ ITF dyes the CSF a fluorescent green that is visible in both white and blue light, and it is a useful adjunct for endoscopic endonasal repair of CSF leaks, with a reported success rate as high as $96 \%$ in previous studies. ${ }^{15-18}$ Additionally, the Trendelenburg position and Valsalva maneuver have been used to promote visualization of fluorescein for CSF leakage from the skull base under the endoscopic endonasal approach. ${ }^{14}$

In the present case, we used the reverse Trendelenburg position and Valsalva maneuver after primary repair to confirm the absence of leakage of visible fluorescent green CSF under microscopic inspection. Nevertheless, the use of ITF has been limited historically because of safety concerns. ${ }^{14}$ Uncommon but severe side effects associated with ITF have been reported in the literature ${ }^{19}$ and range from seizures and neurotoxicity ${ }^{20}$ to headache, lower extremity weakness/numbness, and even opisthotonus, ${ }^{21}$ primarily occurring under rapid injection or lack of sufficient dilution and simultaneous intrathecal application of contrast material. ${ }^{22}$ Therefore, premedication with $10 \mathrm{mg}$ of dexamethasone and $50 \mathrm{mg}$ of diphenhydramine before administration of low-dose ITF has significantly reduced the incidence of associated side effects. ${ }^{14}$ In this case, no post-ITF complications occurred, and CSF leakage did not persist after repair. Therefore, we consider low-dose ITF to be an effective tool in ascertaining the quality of closure and identifying the leak site.

An ASM was the initial reason for CSF leakage in this case. It resulted from dural ectasia with sacral bony defect due to erosion. Therefore, repairing the sacral bony defect was important to decrease the recurrence of an ASM. However, the surgical field of the sacral region under the transabdominal approach is deep, which makes it difficult to seal the bony defect perfectly using only the hands. We built a 1:1 model of the sacrum of this patient using the 3D printing technique. We were able to customize the bone cement fragment to be fixed on the sacrum for repair of the bony defect. PMMA is a highly biocompatible alloplastic material with a long history of use in bone reconstruction. ${ }^{23-25}$ The use of 3D medical molds for these applications can be individualized for each patient. Several advantages of the 3D model technique are listed in an earlier study, ${ }^{24}$ including its use in developing a special understanding of bone morphology, supporting accurate and straightforward planning of preoperative plate bending, and improving the accuracy of bone harvesting for reconstruction. As a result of the present case, we applied the 3D printing technique using PMMA bone cement to perfectly repair the sacral defect, which prevented the recurrence of an ASM.

In the previous study, we found that when repairing skull defects with autogenous bone, despite the bony structure fitting well into the defect, bone absorption is still noted in some cases during longterm follow-up. ${ }^{26}$ The theory for bone loss is possibly associated with pulsation of the brain, which may gradually erode with bone reabsorption of the repaired autogenous bone flap. As in our case, the etiology of congenital bone defect is the pulsation of dural ectasia, with pulsation pumping the underlying bone; therefore, repairing sacral defects with 3D-printed PMMA bone not only has the benefit of fitting the defect perfectly but also may prevent future erosion and reabsorption of the repaired bone flap. However, the reliability and effectiveness of this approach must be supported by long-term follow-up of the patient.

\section{Lessons}

An ASM with iatrogenic CSF leakage is rare and notably difficult to repair and reconstruct. Low-dose ITF is a safe and effective method for identifying a leakage site and repairing the defect completely. 3D-printed real-size models help to achieve rigid bony reconstruction and prevent recurrence. In addition, we believe that preoperative MRI is helpful for differentiating ASMs from other causes of a huge pelvic mass, including ovarian cyst.

\section{References}

1. Akan M, Karaca M, Eker $G$, et al. Is polymethylmethacrylate reliable and practical in full-thickness cranial defect reconstructions? J Craniofac Surg. 2011;22(4):1236-1239.

2. Anari S, Waldron M, Carrie S. Delayed absence seizure: a complication of intrathecal fluorescein injection. A case report and literature review. Auris Nasus Larynx. 2007;34(4):515-518.

3. Arnold PM, Teuber J. Marfan syndrome and symptomatic sacral cyst: report of two cases. J Spinal Cord Med. 2013;36(5):499-503. 
4. Banu MA, Kim JH, Shin BJ, et al. Low-dose intrathecal fluorescein and etiology-based graft choice in endoscopic endonasal closure of CSF leaks. Clin Neurol Neurosurg. 2014;116:28-34.

5. Cheng $\mathrm{CH}$, Chuang HY, Lin HL, et al. Surgical results of cranioplasty using three-dimensional printing technology. Clin Neurol Neurosurg. 2018;168:118-123.

6. Eppley BL. Biomechanical testing of alloplastic PMMA cranioplasty materials. J Craniofac Surg. 2005;16(1):140-143.

7. Fattori R, Nienaber CA, Descovich B, et al. Importance of dural ectasia in phenotypic assessment of Marfan's syndrome. Lancet. 1999;354(9182):910-913.

8. Felisati G, Bianchi A, Lozza P, Portaleone S. Italian multicentre study on intrathecal fluorescein for craniosinusal fistulae. Acta Otorhinolaryngol Ital. 2008;28(4):159-163.

9. Gilete-Tejero IJ, Ortega-Martínez M, Mata-Gómez J, et al. Anterior sacral meningocele presenting as intracystic bleeding. Eur Spine J. 2018;27(suppl 3):276-280.

10. Hollenberg AM, Baldwin AL, Mesfin A, Silberstein H. Rupture of giant anterior sacral meningocele in a patient with Marfan syndrome: diagnosis and management. World Neurosurg. 2018; 119:137-141.

11. Jones ME, Reino T, Gnoy A, et al. Identification of intranasal cerebrospinal fluid leaks by topical application with fluorescein dye. Am J Rhinol. 2000;14(2):93-96.

12. Kohler E, Prentice D. Pseudomeningocele induced transient loss of consciousness in Marfan syndrome. Intern Med J. 2010;40(3): 228-230.

13. Marton E, Billeci D, Schiesari E, Longatti P. Transnasal endoscopic repair of cerebrospinal fluid fistulas and encephaloceles: surgical indications and complications. Minim Invasive Neurosurg. 2005; 48(3):175-181.

14. Massimi L, Calisti A, Koutzoglou M, Di Rocco C. Giant anterior sacral meningocele and posterior sagittal approach. Childs Nerv Syst. 2003;19(10-11):722-728.

15. Mesfin A, Ahn NU, Carrino JA, Sponseller PD. Ten-year clinical and imaging follow-up of dural ectasia in adults with Marfan syndrome. Spine J. 2013;13(1):62-67.

16. Paisan GM, Crandall KM, Chen S, et al. Closure of a giant anterior sacral meningocele with an omental flap in a patient with Marfan syndrome: case report. J Neurosurg Spine. 2018:29(2):182-186.

17. Placantonakis DG, Tabaee A, Anand VK, et al. Safety of low-dose intrathecal fluorescein in endoscopic cranial base surgery. Neurosurgery. 2007:61(suppl 3):161-166.

18. Raftopoulos C, Pierard GE, Rétif C, et al. Endoscopic cure of a giant sacral meningocele associated with Marfan's syndrome: case report. Neurosurgery. 1992;30(5):765-768.
19. Sahin N, Genc M, Kasap E, et al. Anterior sacral meningocele masquerading as an ovarian cyst: a rare clinical presentation associated with Marfan syndrome. Clin Pract. 2015;5(2):752.

20. Sanderson JD, Kountakis SE, McMains KC. Endoscopic management of cerebrospinal fluid leaks. Facial Plast Surg. 2009; 25(1):29-37.

21. Senior BA, Jafri K, Benninger M. Safety and efficacy of endoscopic repair of CSF leaks and encephaloceles: a survey of the members of the American Rhinologic Society. Am J Rhinol. 2001;15(1): $21-25$.

22. Shedid D, Roger EP, Benzel EC. Presacral meningocele: diagnosis and treatment. Semin Spine Surg. 2006;18(3):161-167.

23. Sheikhzadeh S, Brockstaedt L, Habermann CR, et al. Dural ectasia in Loeys-Dietz syndrome: comprehensive study of 30 patients with a TGFBR1 or TGFBR2 mutation. Clin Genet. 2014 86(6):545-551.

24. Snyder BJ, Hanieh A, Trott JA, David DJ. Transcranial correction of orbital neurofibromatosis. Plast Reconstr Surg. 1998;102(3): 633-642.

25. Strand RD, Eisenberg HM. Anterior sacral meningocele in association with Marfan's syndrome. Radiology. 1971;99(3): 653-654.

26. Wolf G, Greistorfer K, Stammberger H. Endoscopic detection of cerebrospinal fluid fistulas with a fluorescence technique. Report of experiences with over 925 cases. Article in German. Laryngorhinootologie. 1997;76(10):588-594.

\section{Disclosures}

The authors report no conflict of interest concerning the materials or methods used in this study or the findings specified in this paper.

\section{Author Contributions}

Conception and design: Wu, YC Yeh, Lin, Hsieh. Acquisition of data: all authors. Analysis and interpretation of data: $\mathrm{Wu}, \mathrm{YC}$ Yeh, $\mathrm{CH}$ Yeh. Drafting the article: $\mathrm{Wu}, \mathrm{YC}$ Yeh, Lin. Critically revising the article: $\mathrm{Wu}$, YC Yeh. Reviewed submitted version of manuscript: Wu, YC Yeh, Lin, $\mathrm{CH}$ Yeh. Approved the final version of the manuscript on behalf of all authors: Wu. Statistical analysis: Wu. Administrative/technical/material support: Wu. Study supervision: Wu, Hsieh.

\section{Correspondence}

Chieh-Tsai Wu: Chang Gung Memorial Hospital, Chang Gung University College of Medicine, Taoyuan City, Taiwan. woodie2@cgmh. org.tw. 